\title{
Proceso de psicoterapia cognitiva posracionalista: Experiencia de adolescentes con desregulación emocional
}

\section{Post-rationalist cognitive psychotherapy process: Experience in adolescents with emotional dysregulation}

\author{
Bárbara Cerda Aedo¹, Verónica Cerda Mora², Priscila Godoy Sarmiento³, Massiel Venegas \\ Hernández ${ }^{4}$, Ismael Morales Ojeda ${ }^{5}$
}

\begin{abstract}
RESUMEN
Objetivo: Comprender la experiencia de adolescentes con desregulación emocional en un proceso de psicoterapia cognitiva post-racionalista. Metodología: Enfoque cualitativo, adoptando una perspectiva fenomenológica. Se busca generar una teoría fundamentada a través de una hipótesis comprensiva desde el modelo teórico cognitivo post-racionalista (Krause, 1995). Resultados: Se pudieron observar cambios significativos en las pacientes intervenidas con respecto a los principales indicadores de desregulación emocional que se dieron a conocer durante las primeras sesiones, verbalizadas tanto por los pacientes como la información obtenida del establecimiento del cual fueron derivadas. Se consiguió que las adolescentes experienciaran las emociones, al nivel de comprender el sentido de continuidad que mantenían y como este se ve alterado al momento de volverse un observador de ellas mismas. Conclusiones: Se pudo acompañar al adolescente en este proceso, vivenciando y conociendo los diferentes aspectos que conllevan el realizar una terapia, tanto a nivel profesional como a nivel personal.
\end{abstract}

Palabras clave: Adolescencia, psicoterapia, cognitivo postracionalista, desregulación emocional.

\begin{abstract}
Objective: To understand the experience of adolescents with emotional dysregulation in a postrationalist cognitive psychotherapy process. Methodology: Qualitative approach, adopting a phenomenological perspective. It seeks to generate a grounded theory through a comprehensive hypothesis from the post-rationalist cognitive theoretical model (Krause, 1995). Results: Significant changes could be observed in the operated patients with respect to the main indicators of emotional dysregulation that were made known during the first sessions, verbalized both by the patients and the information obtained from the establishment from which they were derived. The adolescents were able to experience emotions, at the level of understanding the sense of continuity they maintained and how this is altered when they become an observer of themselves. Conclusions: It was possible to accompany the adolescent in this process, experiencing and knowing the different aspects involved in carrying out a therapy, both professionally and personally.
\end{abstract}

Keywords: Adolescence, psychotherapy, post-translational cognitive, emotional dysregulation.

${ }^{1}$ Universidad Adventista de Chile, Chillán, Chile

Orcid ID: 0000-0001-5872-7382

2Universidad Adventista de Chile, Chillán, Chile

Orcid ID: 0000-0003-3521-2110

${ }^{3}$ Corporación Llequén/ Universidad Adventista de Chile, Chillán, Chile

Orcid ID: 0000-0003-1510-2918

${ }^{4}$ Universidad Adventista de Chile, Chillán, Chile

Orcid ID: 0000-0002-8382-9038

${ }^{5}$ Universidad Adventista de Chile, Chillán, Chile

Orcid ID: 0000-0002-1752-7023 


\section{INTRODUCCIÓN}

El comienzo de la psicología estuvo marcado por importantes aportes al estudio del comportamiento humano, desarrollando diferentes escuelas según el contexto en el cual se encontraban inmersos los profesionales del área de la salud mental. Así fue, que tras muchas corrientes que señalaban las formas en que se podía estudiar al sujeto, en 1990 Guidano (Torrente y Harf, 1995) desarrolla una escuela que centraba su atención en la experenciación de las emociones, para así dar sentido de continuidad a la experiencia. Para este propósito el modelo cognitivo post-racionalista establece que los procesos psicopatológicos tienen su origen en un desbalance de tipo emocional, haciendo hincapié fundamentalmente en el desarrollo emocional de los niños y adolescentes con sus cuidadores (Pena y Oliveira, 2015). Este vínculo es un generador de seguridad, por ende, cuya pérdida o amenaza producirá ansiedad. Este vínculo denominado apego tiene un impacto significativo en el desarrollo y conducta del niño o adolescente pudiéndose modificar desde el nacimiento hasta los 18 años (Crittenden, 2006). Hasta la hora son tres tipos de apegos los que se conocen; seguro, coercitivo y evitativo, donde el seguro enmarca a los niños que buscan y reciben protección, seguridad y apoyo; el coercitivo coloca al afecto como fuente central de información omitiendo la cognición que no le ayuda a protegerse, y finalmente evitativo, donde hay un uso de predicciones temporales de información, que pueden ser verdaderas o pueden estar distorsionadas (Harcha, 2012) Cabe destacar que cada tipo cuenta con subtipos para categorizar más exactamente el desarrollado del niño.

Otro punto que debe tomarse en consideración es el tipo de procesamiento que presenta cada paciente, clasificándolo en normal, neurótico y psicótico (León y Tamayo, 2011).

Finalmente, es importante comprender el sistema del ordenamiento de la experiencia inmediata, el que se caracteriza por una personal y única manera de modificar emociones básicas según el significado que cada individuo da a su experiencia (Naranjo et al., 2010),definiéndose como organización de significado personal (OSP) la OSP dápica, que no presenta polaridades emocionales definidas, fóbica donde predomina la emoción de miedo, y depresiva, donde predomina la tristeza y obsesiva, donde se mantiene cuestionando todo constantemente en búsqueda de la certeza absoluta (Marini et al., 2017)

Por lo tanto, la presente investigación tuvo como objetivo comprender la experiencia de adolescentes que cursan tercero y cuarto año medio en el Instituto Técnico Mabel Condermarin Grimberg, Chillán, que presentan desregulación emocional mediante un proceso de psicoterapia cognitiva post-racionalista. Con esta investigación se desea dejar un precedente para realizar futuros estudios basándose en la teoría de las emociones, mediante la psicoterapia cognitivo post-racionalista.

\section{METODOLOGÍA}

Los datos que permitieron realizar esta investigación fueron de corte cualitativo con método inductivo, esto desarrollándose bajo el paradigma exploratorio fenomenológico. La recolección de información será mediante análisis de caso.

Las participantes del estudio fueron alumnas del liceo técnico profesional particular subvencionado de la ciudad de Chillán, pertenecientes a los cursos de 3ero y 4 to medio de escolaridad diurna, desde donde fueron derivadas.

Las 5 participantes de la investigación fueron estudiantes entre 16 y 19 años, que se encuentran cursando enseñanza media en el liceo Técnico Mabel Condemarin Grimberg. El sexo de las participantes es femenino y todas son de nacionalidad Chilena. Es importante saber que cada una de las participantes manifestó algún tipo de desregulación emocional durante los pasados 6 meses. Las participantes fueron seleccionadas mediante un muestreo intencional o de conveniencia, ya que se realizó a través de la psicóloga del establecimiento, quien identificó presencia de desregulación emocional en ciertas alumnas y ante esto se llevó a cabo la selección.

Posteriormente se realizó una reunión con los padres de las participantes para explicarles en qué consistía el estudio y se les solicitó firmar el consentimiento informado para poder llevar a cabo el proceso psicoterapéutico.

La investigación se extendió por 12 semanas, con 1 a 2 sesiones por semana, dependiendo 
de la disponibilidad horaria de las pacientes y se realizó en una de las dependencias del Centro Médico Libertad Ubicado en Av. Libertad, en la cuidad de Chillán. La técnica de recolección de datos utilizada fue la observación participante. El principal instrumento que se utilizara para generar datos será la entrevista psicológica semiestructurada clínica, a través de las siguientes fichas y pautas y mediante la técnica clave de la terapia postracionalista, que es la Moviola. Los instrumentos fueron:

Ficha Clínica: Ficha donde se ingresaron todos los avances y datos adquiridos por los pacientes en cada sesión, además de los antecedentes personales y relevantes, dejando registro de cada experiencia emocional vivenciada por parte del individuo en su proceso terapéutico. Se estimó que esta herramienta permitiría acceder, en un ambiente protegido, a la experiencia vivenciada por el paciente y que significado le atribuye a la terapia.

Pauta de Supervisión de Casos: Pauta que permite integrar los datos obtenidos en la ficha clínica de una manera ordenada y práctica que facilita la realización de hipótesis diagnósticas.

Pauta de Condensación: Instrumento que permite la realización de un resumen por cada caso, donde se destacan principalmente los siguientes componentes: apego, tipo de procesamiento, OSP. Consiste en seleccionar las verbalizaciones y hechos recogidos en sesión, que indiquen algún tipo de apego, tipo de procesamiento, OSP, ya anteriormente mencionadas, posteriormente se conectan con la que correspondan permitiendo un bosquejo claro del diagnóstico.

Pauta Registro Intervenciones: Pauta que consiste en los datos principales de cada paciente, además del registro de lo ocurrido sesión a sesión durante las intervenciones. Su principal función es tener de manera ordenada y clara todo lo ocurrido durante cada sesión para posteriormente realizar un análisis más completo.

Moviola: Esta técnica representa la interfaz con la realidad clínica de una teoría que la sustenta y la vuelve coherente, siendo parte de un método en el cual se articulan y se tornan explícitas las maneras en las cuales puede tomar forma una aproximación terapéutica.
Esta investigación como es de carácter cualitativa no analiza por lo tanto datos estadísticos, sino que procesos que van desarrollando la psicoterapia a través de los diferentes procedimientos terapéuticos. $Y$ estos procedimientos se establecen como etapas que se deben cumplir y respetar para el funcionamiento y desarrollo funcional de la psicoterapia.

Para asegurar la confidencialidad de los datos, no se utilizaron los nombres de las pacientes otorgándoles un número durante la investigación para referirnos a ellas. Las participantes firmaron un asentimiento informado, previa participación en el estudio. Se solicitó autorización a los apoderados responsables a través de consentimiento informado autorizado por el Comité de Bioética, además del dictamen del Comité de Bioética de la Universidad Adventista de Chile sobre proyectos de investigación que utilicen datos personales.

\section{RESULTADOS}

Para la presentación de los resultados e interpretación de las entrevistas semiestructuradas clínicas, haremos el análisis desde la individualización de cada paciente y de los avances obtenidos en cada sesión.

\section{Paciente $\mathbf{N}^{\circ} \mathbf{1}$}

La paciente $\mathrm{N}^{\circ} 1$ es una adolescente de 19 años, quien presenta un desarrollo físico y cognitivo acorde a su etapa de desarrollo evolutivo, cursando 4to año de enseñanza media, con especialización en el área de educación parvularia. Nació de una relación matrimonial entre sus padres, con quien no tiene mucho contacto. Ella se independiza al salir del Servicio Nacional de Menores (SENAME), al cual ingreso tras haber sido víctima de abuso sexual por parte de su hermano 5 años mayor que ella. Posterior a la develación del abuso, la paciente se entera que por parte de su familia materna no dan credibilidad al relato.

La familia nuclear de la paciente estaría conformada por su pareja de 20 años de edad, quien no ha terminado sus estudios en la enseñanza media, y estaría trabajando como 
vendedor por internet de un negocio creado por la paciente para generar recursos económicos con la finalidad de suplir las necesidades básicas de su hija de 2 años de edad.

En torno a ello, se visualiza un estilo de crianza orientado en lo negligente, presentando ausencia afectiva, además de no ser cubiertas sus necesidades básicas, teniendo que ser remitida a un hogar para resguardar sus derechos y velar por su bienestar. Además de la presencia de un patrón de apego evitante, el cual se ve reflejado en la constante incertidumbre que se ve envuelta la dinámica existente entre madrehija, al no haber tenido nunca una sensación de resguardo o protección por parte de su adulto significativo.

Es importante destacar además que el tipo de procesamiento que presenta la paciente $\mathrm{N}^{\circ} 1$ durante las intervenciones es neurótico; al no poder comprender la consigna de la técnica de la moviola, al igual que al momento de responder de manera vaga a las preguntas que se le realizaron durante el proceso de psicoterapia. Este tipo de procesamiento podría ser comprendido debido a la presencia de sintomatología depresiva, como desánimo, disminución del apetito sexual, anhedonia, desmotivación, hipersomnia, pérdida de apetito entre otras; producto de una posible psicopatología depresiva mayor.

Por otra parte la paciente $\mathrm{N}^{\circ} 1$ presenta conductas que indicarían la presencia de una OSP depresiva con cierre fóbico, al verbalizar que "se siente sin ganas de hacer nada, como desanimada", al igual que menciona que "me he notado más irritable, me di cuenta de eso cuando le grite a mi hija, y ella me miro asustada y luego se puso a reír, no sé qué me pasa", "hay momentos en los que no me dan ganas de hacer nada, solo quiero estar acostada con los ojos cerrados sin luz", y así varias ocasiones en las que la paciente $N^{\circ} 1$ refiere sentirse triste 0 con rabia debido a las situaciones por las que ha tenido que pasar. El cierre fóbico se puede observar, en las constantes pesadillas y crisis de pánico que presenta la adolescente frente ciertas situaciones que le generan estrés. Es mediante esto que se realiza un diagnóstico de OSP como una organización depresivo con cierre fóbico.

Se pudo concluir que la presencia de desregulación emocional en la adolescente es una respuesta a los conflictos que se han generado a nivel familiar, debido al abuso sexual sufrido durante su infancia, y las consecuencias en la reestructuración que se dio a causa de esta develación. La sintomatología vuelve a surgir debido al contacto con su hermano mayor quien al volver a la casa, provoca en la paciente el conectarse con la experiencia de vida que consideraba ya superada; cambiando la tonalidad emotiva por la cual estaría pasando la adolescente, lo que se manifestó en recuerdos y pesadillas vívidas que alteraron la experiencia inmediata, causando una interrupción en el sentido de continuidad que la paciente habría logrado generar después de este suceso traumático. Es a partir de estos eventos significativos que se procede a recabar información, no logrando llegar a término con el proceso terapéutico debido a las constantes ausencias por parte de la paciente a las sesiones.

\section{Paciente $\mathrm{N}^{\circ}$ 2:}

La paciente $\mathrm{N}^{\circ} 2$ es una adolescente de 17 años, quien presenta un desarrollo físico y cognitivo acorde a su etapa de desarrollo evolutivo, cursando 3er año de enseñanza media, con especialización en el área de educación parvularia.

Nace producto de la filiación matrimonial entre sus progenitores; falleciendo su padre a los 52 años, producto de un asesinato perpetrado por un esquizofrénico en Santiago, teniendo en ese entonces 10 años.

Con respecto a la relación que tiene con su progenitora la paciente refiere que desde el fallecimiento de su padre, esta cambió, señalando que desde ese momento comenzó a quedar sola en casa, cosa que nunca sucedía antes, porque su padre nunca dejaba que eso pasara. Desde ese momento comienzan a presentarse estos sentimientos de soledad que prevalecen durante un largo tiempo.

La adolescente al comenzar a experimentar estos sentimientos de soledad, busca refugio en la casa de sus primos, donde comienza a pasar más tiempo con ellos y a sentirse parte de la familia; hasta que ocurre una infidelidad por parte de su tío, y las peleas comienzan a ser constantes en la casa. La paciente refiere que para ella esta situación no era común, y la llenaba de ansiedad, el tener que escuchar a 
sus tíos pelear de manera verbal al comienzo, pasando luego al maltrato físico. Es en este contexto cuando comienza a presentar sintomatología ansiosa, a través de dolor de estómago y cabeza.

Posterior a estas peleas e incidentes, surge un conflicto en la escuela donde asiste la adolescente junto a su prima, creándose rivalidad entre ambas con respecto a quien mantenía más relaciones de amistad. Frente a esta situación se realiza una denuncia, por acoso a través de Facebook por parte de una de sus compañeras a su prima, siendo la paciente señalada como la responsable de estos mensajes. Al llegar a instancias superior se resuelve el asunto, dejando una brecha bastante amplia y negativa entre las relaciones existentes con la familia por línea materna y la paciente.

Se observa en la paciente que la desregulación emocional que presenta es una respuesta al conflicto presente con su madre, al no poder verbalizar o expresar sus emociones hacia ella, guardando cada emoción y canalizándola a través de la somatización. De igual manera para regular sus niveles de ansiedad es que recurre a la tricotilomanía, claro registro de la impulsividad que presenta la paciente, al igual que la ansiedad observada durante las sesiones y la constante necesidad de querer sentirse validada por sus pares y familia, mostrando una OSP depresiva con cierre fóbico, al ser para ella importante el como los demás se siente en función a su actuar; llegando como resultado la represión de todas las conductas que podrían según ella generar alguna incomodidad en el resto, despreocupándose de su sentir; funcionando en fin de los otros, dejando de lado su experiencia de vida con el fin de lograr una relación armoniosa.

un principio con la paciente $\mathrm{N}^{\circ} 2$, se generó una adecuada vinculación terapéutico, asistiendo a la mayoría de las sesiones pactadas, y justificando su ausencia frente a las cuales no podía asistir. No hubo inconvenientes en la utilización de la técnica de la moviola, mediante la cual se trabajaron las principales manifestaciones de desregulación emocional, (depresión, impulsividad y ansiedad).

La psicoterapia consistió en la asistencia a sesiones de manera semanal, con el objetivo de revisar cuales eran las experiencias de vida de la paciente, además de experienciar cuáles eran las emociones que predominaban en ese momento, a través de la técnica de la moviola.

La materia prima con la cual se trabajó durante las 7 sesiones a las cuales asistió la paciente, fueron las experiencias de vida generadas entorno a la soledad y los sentimientos de tristeza, rabia y miedo, expresados por la adolescente.

Se trabajó con los eventos significativos seleccionado por la paciente, tales como las peleas que tenían sus tíos, las burlas por parte de su mamá y hermana al verla sin pelo, el proceso de duelo por parte del fallecimiento de su papá, la sensación de soledad y desprotección que vivió en los meses posteriores a la muerte de su papá.

Es a partir de estos eventos significativos que se procede a recabar información, logrando llegar a término con el proceso terapéutico, observando cambios significativos en la paciente, tales como la disminución de la impulsividad en función de la tricotilomanía, y el reconocer la tonalidad emotiva de cada evento significativo de la experiencia inmediata de la adolescente. Durante el proceso la paciente logra hacer consciente su manera de ser, sentir y funcionar, internalizando las experiencias de soledad e impulsividad a su personalidad.

\section{Paciente $\mathbf{N}^{\circ}$ 3:}

La adolescente fue derivada debido a conflictos y problemas de conductas con los docentes y compañeros, presentando desacuerdos y peleas constantes, además de haber sufrido una leve parálisis en el rostro, asociado a una posible crisis de pánico no diagnosticada.

Se observó en la paciente que la desregulación emocional que presenta es una respuesta a sus conflictos con su familia, especialmente por la falta de comunicación y de apoyo por parte de estos, ya que asegura que desde que nació su hermana pequeña, ha quedado vulnerable y sola. Otra de las características que se encuentran en la adolescente es el descontrol de impulsos tanto a nivel personal como con el grupo de pares y con la autoridad. Esto se desencadena en conductas de rebeldía y confrontación especialmente en el contexto educacional, aunque presenta buenas calificaciones y buena 
disposición en las asignaturas. El enojo y los episodios de ira son una variable regular dentro de su dinámica, mencionando que no puede tener una conversación normal con sus padres y menos con sus profesores. También se observa que no puede conectarse con sus emociones, asegurando que es una persona fría y que no le importan los demás. Esto se relaciona con el estilo de apego que presenta, denominado evitante, ya que desde que era pequeña sus padres la han tratado con frialdad y lejanía. Su OSP se presentan como depresiva caracterizado por sentimiento de soledad y abatimiento, y por tener una imagen de sí misma desvalorada y negativa. Otro indicador de la presencia de desregulación emocional, son las autolesiones, aunque se produjeron en un breve periodo de tiempo, esto también fue un indicador de angustia y de situaciones conflictivas tanto en su vida personal como con sus familiares.

Es a partir de estos eventos significativos que se procede a recabar información, logrando llegar a término con el proceso terapéutico, observando cambios significativos en la paciente, tales como la disminución de los niveles de ansiedad, agresividad y la presencia de mayor contacto con su figura paterna, y el reconocer la tonalidad emotiva de cada evento significativo de la experiencia inmediata de la adolescente. Durante el proceso la paciente logra hacer consciente su manera de ser, sentir y funcionar, internalizando las experiencias de ansiedad, impulsividad y agresividad a su personalidad.

\section{Paciente $\mathbf{N}^{\circ}$ 4:}

La paciente $\mathrm{N}^{\circ} 4$ es una adolescente de 16 años, quien presenta un desarrollo físico acorde al esperado para la etapa del desarrollo cursada, consigna escolaridad de 3er año de enseñanza media. Según antecedentes con los que se cuenta, presenta una historia vital en donde se han presentado diversos problemas familiares de larga data, primordialmente asociados a maltrato físico por parte de su familia hacia la paciente.

Entorno a ello, se visualiza un estilo de crianza orientado a lo permisivo, con presencia de inadecuados canales de comunicación, con una manipulación activa en la relación con los otros, para conseguir sus objetivos y necesidades, además de la presencia de un patrón de apego Coercitivo, C5- Punitivo. En el discurso del adolescente, existe la demanda constante de aceptación por parte de otros. Es importante destacar que el tipo de procesamiento que presenta la paciente $\mathrm{N}^{\circ}$ 4 durante las intervenciones es el denominado psicótico. Se observa principalmente en la presencia de malas calificaciones, luego en la incapacidad de comprender las consignas al repetirlas reiteradas veces, como en el caso de la utilización de la técnica de la moviola. La rigidez de su pensamiento se hace presente en la ausencia de consciencia de algunos hechos, por ejemplo, su deseo de ser madre a los 16 años, acompañados de las siguientes frases "Si nace enfermo, pido unas horas al liceo para poder ir a cuidarlo", "mi mamá se hará cargo mientras estudio", "si se cómo ser madre, mi mamá me ha explicado cómo se debe cuidar a un bebé". Un acontecimiento que es necesario destacar, es la incapacidad de llegar a la hora asignada a las intervenciones. Su horario se encontraba alternado entre las 15:00 horas un día y el siguiente las 16:00 hr, pero la paciente $\mathrm{N}^{\circ} 4$ continuaba llegando en el horario de las 15:00 $\mathrm{h}$ aunque se le recordara su horario para la siguiente sesión.

Se observa la desregulación emocional por parte de la paciente, desde su infancia, en donde desde pequeña ha recibido castigo físico por parte de sus figuras significativas, además de concientizarla constantemente de que los problemas ocurridos en el hogar eran culpa de ella y sus conductas. Esto fue desarrollando en la paciente conductas oposicionistas que se hacían presentes en el hogar, sobre todo con sus padres además de constantes manifestaciones de desborde emocional. Esta relación con sus figuras de apego es la que le da forma al apego coercitivo presente en la evaluada, observándose principalmente en su manera de relacionarse con su grupo de pares, manteniendo distancia física total y contacto únicamente a través de redes sociales. También se puede explicar la desregulación emocional en el bullying sufrido durante toda su niñez por parte de su grupo curso y familia, lo que desencadenó en la paciente maneras agresivas e impulsivas de expresar sus emociones. Por otro lado, la paciente $\mathrm{N}^{\circ} 4$ presenta conductas que indican una OSP dápica, como son la presencia de vestimenta llamativa durante todas las intervenciones, además de mantener una variedad de relaciones de duración aproximada de un mes con personas conocidas a través 
de la aplicación de comunicación instantánea WhatsApp sumándose la realización de autolesiones, tras las rupturas en sus relaciones para posteriormente publicarlas en las redes sociales. Durante su discurso se hace presente también verbalizaciones que señalan una organización dápica, por ejemplo: "le digo que si para que no se aleje", "no sé si quiero, pero él quiere", "¿Qué van a pensar de mí?" Además, se observan algunos hechos en conjunto con verbalizaciones que impresionan de una OSP depresiva, como son: la constante culpa de las peleas de sus papás por sus notas; verbalizaciones tales como "el mundo sería mejor si desapareciera", "a veces quiero morirme", "creo que no sirvo para nada". Es mediante esto que se realiza un diagnóstico de OSP como una organización dápica con cierre depresivo.

Dentro de la línea motivacional, el adolescente presenta escasos intereses sistemáticos por actividades, planteándose incluso una escasa orientación a establecer lazos sociales con pares o desarrollar un sentido de pertenencia, con un grupo humano de pares.

En lo concerniente al desarrollo intelectual, se aprecia un repertorio bajo de vocablos, el que tiende a vincularse a aspectos más evidentes y operativos de la realidad.

Se aprecian alteraciones sobre el despliegue de capacidades de atención y concentración al igual que a nivel cognitivo. No obstante, debe tenerse en consideración una clara influencia del componente emocional, sobre los rendimientos o funciones intelectuales antes señaladas.

Desde el inicio con la paciente $\mathrm{N}^{\circ} 4$, la técnica utilizada fue la moviola, mediante la cual se trabajaron las principales manifestaciones de desregulación emocional, (agresividad, conductas autolesivas, ansiedad y conductas oposicionistas).

Al terminar las sesiones, después de un avance significativo con la paciente $\mathrm{N}^{\circ} 4$ donde disminuyeron significativamente las conductas oposicionistas, y las autolesiones ya no eran la primera opción frente a los problemas, siendo capaz de visualizar otras formas, se realizan los trámites para derivarla al equipo de salud mental del Centro de Salud Familiar (CESFAM) al que pertenece para poder continuar con psicoterapia.
La culpa fue otro avance importante en el proceso psicoterapéutico, debido que al finalizar las intervenciones ya no estaba presente, siendo la paciente capaz de ver los hechos con una perspectiva diferente.

Se observaron cambios significativos en la paciente, tales como la disminución de las conductas oposicionistas y las autolesiones (agresividad) y el reconocer la tonalidad emotiva de cada evento significativo de la experiencia inmediata de la adolescente. Durante el proceso la paciente logra hacer consciente su manera de ser, sentir y funcionar, internalizando las experiencias de agresividad e impulsividad a su personalidad.

\section{Paciente $\mathrm{N}^{\circ}$ 5:}

Paciente $\mathrm{N}^{\circ} 5$ es una adolescente de 17 años, quien presenta un desarrollo físico acorde al esperado para la etapa del desarrollo cursada, consigna escolaridad de 3er año de enseñanza media. Vive con su abuela y sus dos hermanas menores, debido a que su madre fallece hace 5 años.

Nace producto de una relación pasajera entre sus progenitores, siendo abandonada por el padre y sufriendo maltrato físico por parte de su madre, maltrato que culminó con un incidente en donde debido a retiradas patadas proporcionadas por su progenitora, le produce un daño en el estómago a la edad de 6 años.

Según antecedentes con los que se cuenta, paciente $\mathrm{N}^{\circ} 5$ presenta una historia vital de maltrato físico y abandono, además de haber sido víctima de abuso sexual a la edad de 5 años por parte de un "amigo" que conoció en el bar, que el padre de sus otras dos hermanas la llevaba. Es en un momento de descuido mientras ella duerme en un sillón en la entrada, que el ofrece jugar con ella, posteriormente la lleva a una habitación oscura donde ocurre el hecho. Luego de esto paciente pide ayuda siendo llevada a constatar lesiones y terminando el agresor con una condena por un periodo de 10 años.

En torno a ello, se visualiza un estilo de crianza orientado a lo autoritario, con presencia de inadecuados canales de comunicación, maltrato físico y psicológico, además de abandonos 
reiterados por parte de su madre. Se hace presente además un patrón de apego A1,2Inhibido/socialmente fácil. En el discurso del adolescente, existe constantemente presencia de culpa, por todo lo que le ha ocurrido, además del rencor hacia su madre.

Es importante destacar que el tipo de procesamiento que presenta la paciente durante las intervenciones es el denominado normal. Se observa principalmente en la presencia de excelentes calificaciones, la capacidad de comprender las consignas de manera inmediata, como en el caso de la utilización de la técnica de la moviola. La flexibilidad de su pensamiento se hace presente en la capacidad de seguir manteniendo el control de su mente, incluso en momentos donde bordea lo psicótico, por ejemplo, frente a su deseo de quitarse la vida refiere haber visto en su mente como ella tomaba un cuchillo y se mataba, es en ese momento que a pesar de toda la perturbación emocional que sentía refiere que se va a dormir con su abuela para evitar llevar a cabo la acción, o frente a los episodios psicóticos, tenía la habilidad para distinguir que estos "estaban fuera de su mente", por lo que "no les temía, porque sabía que no existían en la realidad".

Por otro lado, paciente $\mathrm{N}^{\circ} 5$ presenta conductas que indican una OSP depresiva, como son la ideación suicida que presentó en dos ocasiones, o cuando señala que aún guarda rencor a su madre por no haberla criado a ella, pero poder haber criado a su hermana que le sigue en edad. Se observa en los sentimientos de culpa constantes, como cuando refería "que se merece todo lo que está viviendo", "que si la golpeaban era por ser muy desordenada", "que esto que le ocurre es el Karma y no hay más remedio que aceptarlo", y principalmente, el sentimiento de tristeza (que en muchas ocasiones se observó a través de la ira).

Por otra parte, se observan algunos hechos en conjunto con verbalizaciones que impresionan de una OSP dápica; por ejemplo cuando refiere "vomitar en momentos de estrés. Puede ser después de comer, o ante situaciones que le generen mucha ansiedad. No entiende el porqué, solo vomito", o la verbalización de frases como "me preocupo mucho por lo que piensen de mí", "importa más como me ven que como me siento", "no consigo conectarme con mis emociones", "pospongo mis cosas por estar para el resto".
Es mediante esto que se realiza un diagnóstico de OSP como una organización depresiva con cierre dápica.

Uno de los hechos más importantes trabajados con la técnica de la Moviola fue la enfermedad de su hermana menor, motivo principal por el que está sacando el título técnico de enfermería. Desde pequeña, la paciente refiere haber pasado noches en el hospital con su hermana, actualmente es probable que necesite trasplante de riñón, lo que la tiene muy preocupada, y no solo por eso, si no por el ofrecimiento de la pediatra de adoptarla en caso de que fallezca su abuela con quien vive. Es aquí donde explica que le duele mucho pensar en eso, pero que es la mejor opción para asegurar el buen vivir de su hermana menor.

En este proceso se concluye que la dificultad de conocer y regular sus emociones por parte de la participante, se debe a que desde su corta edad fue formando vínculos evitantes con sus principales figuras de apego, empezando por su madre, de quien recibía maltrato físico, para continuar con la pareja de su abuelo quien de la misma forma que su madre era violenta con ella.

Al terminar las sesiones, se observa que no hay avances significativos esperados, siendo el único progreso observable el que su nivel de consciencia haya aumentado al punto de crear en ella un sentido de discontinuidad en su experiencia emocional, al ser capaz de conocer cómo funciona y la forma en la que manejaba sus emociones.

Al perder el sentido de continuidad y ver que las respuestas que se daban para su forma de ser no eran las correctas, comienza un aumento de los episodios psicóticos manifestándose en gran manera rasgos límites de personalidad.

Se realizan los trámites para derivarla al equipo de salud mental del CESFAM al que pertenece para poder continuar con psicoterapia y obtener una derivación a psiquiatra.

Es a partir de estos eventos significativos que se procede a recabar información, logrando llegar a término con el proceso terapéutico, observando cambios significativos en la paciente, tales como la disminución de la impulsividad, y agresividad (autolesiones) y el reconocer la tonalidad emotiva de cada evento significativo de la experiencia inmediata de la adolescente. Durante el proceso 
la paciente logra hacer consciente su manera de ser, sentir y funcionar, internalizando las experiencias de agresividad e impulsividad a su personalidad. Cabe mencionar que, tras acabar con todas las sesiones, la paciente ya había logrado bajar la intensidad de los episodios psicóticos, episodios que con la intervención de algún médico psiquiatra podría llegan a su fin.

En la siguiente tabla se resumen los diagnósticos de cada una de las pacientes trabajadas

Tabla 1

Psicodiagnóstico realizado a las adolescentes con desregulación emocional luego de un proceso de psicoterapia cognitiva post-racionalista

\begin{tabular}{llll} 
Paciente & Apego & Tijpo de procesamiento & OSP \\
№1 & Evitante & Neurótico & Depresiva con cierre fóbico \\
№2 & Evitante & Normal & Depresiva con cierre fóbico \\
№3 & Evitante & Normal & Depresiva con cierre dápico \\
№4 & Coercitivo, C5-Punitivo & Psicótico & Dápico con cierre depresivo \\
№5 & Evitante, A1,2-Inhibido/socialmente fácil & Normal & Depresiva con cierre dápico \\
\hline
\end{tabular}

\section{DISCUSIÓN}

La adolescencia y la desregulación emocional son dos variables que se correlacionan de manera significativa frente a la incapacidad de la mayoría de los adolescentes para tener un buen control sobre sus emociones, dificultándoseles el poder desenvolverse de manera adaptativa frente a su grupo de pares.

Como es sabido, en la adolescencia el grupo de amigos o pares se vuelve una parte muy importante para el joven en su desarrollo psicosocial. "Es de suma relevancia que la amistad en la adolescencia sea positiva para el joven, a fin de que su desarrollo transcurra de la forma más sana posible" (Méndez, 2012).

Frente a esto se vuelve visible la importancia de realizar una investigación que permita conocer de manera directa y desde la propia subjetividad del paciente su manera de relacionarse y como esta se ve afectada por sus manifestaciones emocionales desreguladas, por ende, es que la presente investigación se planteó por objetivo conocer la experiencia de un grupo de alumnas que manifestaban desregulación emocional desde el enfoque cognitivo post-racionalista y que, a diferencia de otras investigaciones, al ser cualitativa permitió la obtención de información más completa sobre la manera en que se experimentaban las emociones, dando pie para así conocer el mundo subjetivo del paciente a mayor profundidad, facilitando la inmersión de cada una de las investigadoras en la vida e historia del paciente en cuestión. Es por esto que posee algo exclusivo en relación a otras investigaciones realizadas sobre la misma temática.

De acuerdo a las hipótesis obtenidas durante este trabajo investigativo (estilos de apego, tipos de procesamiento y OSP) fue posible comprende de una manera íntegra la forma en que las pacientes experimentaban su emocionalidad, mantienen su coherencia interna y su autoorganización. Todo esto permite su adaptabilidad al medio que las rodea tratando constantemente de alcanzar la mismidad, sin darse cuenta de que, en varias ocasiones, incongruencias en su coherencia interna las llevan a este estado de ipseidad o patología.

La base para este tipo de análisis es la que se ha mencionado y discutido durante todo el proceso investigativo, la teoría cognitiva postracionalista. Es en esta corriente donde en efecto, el observador ya no aparece en esta posición de privilegio observando objetivamente las cosas tal como son; más bien con su observación, el observador introduce un orden en lo que observa, y lo que observa es más dependiente de su estructura perceptiva que de algo externo a él. "Lo que se empieza a notar claramente que el mundo de regularidades que vivenciamos es construido por cada observador". (Ruiz, 2007) 
Durante el trabajo investigativo un factor en común entre las adolescentes fue la OSP depresiva (ya sea como principal o como cierre). Principalmente se observó en su manera de ver el mundo, de teñir con la emocionalidad de tristeza las interacciones y dificultades que se hacían presente día a día dándole sentido de continuidad a su experiencia inmediata, con una explicación muchas veces, expresada como sentimientos de rabia, pero siempre con un núcleo basado en la tristeza. Básicamente como se menciona en apartados anteriores, fueron adolescentes caracterizada por un sentido ambivalente y dicotómico respecto al todo o nada, incontrolabilidad en el pensamiento y comportamiento. El paciente los percibe como extraño. Necesidad de certeza absoluta (Cayupe, 2013).

Es importante, no solo hacer énfasis en cómo se observó la emocionalidad de tristeza en ellas, si no entender qué de lo central en la experiencia de pérdida.

Reflejara en la diferenciación selectiva, cuando al final de los años los conjuntos de esquemas básicos de los adolescentes estén suficientemente diferenciados y amplificados para fomentar una conceptualización que puede ser ordenado en circuitos oscilante entre polaridades emocionales de tristeza y rabia (Guidano, 1987, citado en Cayupe, 2013), que es la máscara con la que se oculta esta emoción primaria.

Impulsividad, autolesiones, conductas oposicionistas; todas vividas desde el enojo, desde sentimientos de ira, pero basadas en la tonalidad emotiva de la tristeza. Aquí entonces aparece la siguiente pregunta, ¿Cómo pueden comprenderse desde el enojo?, ¿la oposición y autolesiones son manifestaciones de ira?; las conductas impulsivas como ser incapaz de mantener el control ante situaciones, o atacar de manera agresiva a sus pares cuando no compartían el punto de vista de la adolescente solo son las más visibles, pero si se ahonda, nos damos cuenta que las autolesiones, o esta necesidad de hacerse daño responden a sentimientos de incapacidad, poca valía, no merecedoras de afecto, (tristeza) que se traducen en rabia frente a la imposibilidad de tener un control ante dichas situaciones, estos sentimientos generan en la adolescente frustración, lo que posteriormente las lleva al enojo. $O$ en las conductas oposicionistas, como desobedecer deliberadamente, buscar realizar acciones contrarias a las solicitadas nos muestran otra manifestación de ira, donde esta se expresa mediante conductas que, al ser contrarias a las exigidas, denotan al otro que las adolescentes han desobedecido conduciéndolos a discusiones en las que finalmente se terminará por expresar la rabia de manera directa.

Para poder configurar una OSP depresiva, un factor clave es un patrón de apego evitante (León y Tamayo, 2011) generalmente (en algunos casos dependerá de cual sea la OSP principal en el paciente). Este apego puede verse afectado por factores como; perdida de uno de los padres durante la infancia, la experiencia de no haber logrado nunca, a través de las etapas de maduración, un vínculo emocional seguro y estable, a pesar de los continuos esfuerzos en esa dirección y la inversión de la relación padrehijo, en que el niño es hecho responsable por el cuidado de un padre.

Al revisar los casos de las cinco pacientes se ven uno o más de estas características en su patrón de apego, conformando su identidad en base a la emoción de tristeza mediante experiencias de pérdida o afecto.

Este trabajo dio a conocer la manera en la que se puede experienciar una emoción mediante la construcción del hecho significativo, y como esto permite elevar el nivel de consciencia en el paciente para que sea capaz de comprender como si fuera un observador su manera de funcionar y adaptarse al mundo.

Mediante estos resultados se contribuyó al conocimiento previo sobre la terapia y su manera de trabajar, ya que permite obtener una visión más cercana de los efectos de la terapia y su manera de realizarse en adolescentes.

Como grupo de investigación utilizamos para el desarrollo de nuestro estudio la metodología cualitativa, debido a que este enfoque entregaba herramientas que pueden ser utilizadas para variados temas de investigación, no discriminando su aplicación ni desarrollo, adaptándose a lo que deseábamos sin cambiar los contenidos y los procesos a realizar.

Además, al trabajar con una muestra más reducida y específica es posible observar en las adolescentes los cambios significativos que se fueron desarrollando durante la psicoterapia, sin 
modificar ni cambiar el modelo. También cuando se desarrolla la metodología observación participante, esto se refiere a estar inmerso en la investigación y no solamente como un mero espectador, siendo el terapeuta un perturbador emocional estratégicamente orientado durante el proceso psicoterapéutico y participando de manera activa en el desarrollo de la misma.

Debido a esto, el marco teórico se va desarrollando y co-construyendo a medida que la investigación avanza y se descubren nuevos conceptos e ideas que estudiar y analizar.

Como último punto antes de cerrar este apartado, es necesario hacer mención de una observación que se realizó durante el proceso investigativo. Cuando iniciamos el proceso psicoterapéutico las adolescentes se encontraban sin recibir terapia, ni fármacos, ni atención por parte de ningún psicólogo o psiquíatra, aun cuando en sus casos se vislumbraban grandes falencias que interferían en su salud mental.

Frente a esta situación nos hacemos la siguiente pregunta, ¿Cuántas alumnas se encontraran en las mismas situaciones de desregulación emocional en este establecimiento sin recibir atención de salud mental?, y luego, nos cuestionamos, ¿ocurrirá esta situación en todos los colegios de Chillán? Ante esto quedamos sin una respuesta ya que es una información muy difícil de obtener sin una exhaustiva investigación que se dedique a conocer esas cifras.

¿Qué medidas deberían tomarse ante tales situaciones?, entendiendo la prioridad y urgencia de lo que ocurre, y ¿Será un problema de quienes cumplen el rol de psicólogos en los colegios?, ¿o será falta de profesionales de la salud mental trabajando en establecimientos educaciones?

Con respecto a la participación de las estudiantes, cabe destacar que en su mayoría presentaron muy buena adhesión a las intervenciones, aunque algunas presentaron ausencias en ciertas fechas, aun así, se mostraban cooperadoras e impresionando motivadas a asistir. Esto permitió realizar las intervenciones de la mejor manera obteniendo avances que culminaron en procesos significativos. El caso que no manifestó adhesión terapéutica abandonó el proceso luego de la tercera intervención. Si bien la investigadora trabajó vínculo y reencuadre, es la paciente quien decide dejar de asistir completamente. Sin embargo, de las 5 participantes, la paciente numero 3 mantuvo un $100 \%$ de asistencia en sesión, construyendo así experiencias que posteriormente acabaron en un avance significativo en muchas de las manifestaciones de desregulación emocional.

Teniendo en cuenta lo mencionado anteriormente, se podría afirmar que el objetivo principal de esta investigación se cumplió en su totalidad, ya que independiente de las inasistencias o los pocos avances significativos por parte de la paciente $\mathrm{N}^{\circ} 5$, se consiguió que las adolescentes experienciaran las emociones, al nivel de comprender el sentido de continuidad que mantenían y como este se ve alterado al momento de volverse un observador de ellas mismas.

Es a través de la experiencia del terapeuta que se puede concluir que, de acuerdo a los objetivos propuestos referente a la experienciación del paciente, se pudo acompañar al adolescente en este proceso, vivenciando y conociendo los diferentes aspectos que conllevan el realizar una terapia, tanto a nivel profesional como a nivel personal. Se conoció lo que es aplicar la terapia cognitiva post-racionalista, en una población que no ha sido motivo de suficientes estudios sobre el tema tratado, que es la desregulación emocional.

Finalmente durante el proceso de desarrollo y discusión de la investigación, se ha llegado a consignar que las limitaciones durante el estudio son las siguientes: existe una alta probabilidad de que los pacientes al momento de comenzar la psicoterapia no generen adherencia al proceso y cabe la posibilidad además que al momento de pedir el consentimiento informado a los padres o tutores responsables de los estudiantes, para comenzar con el proceso terapéutico, estos no estén de acuerdo en firmar el documento, afectando el número de las participantes de la investigación.

Los resultados obtenidos durante el proceso de investigación dan cuenta del avance con respecto a la literatura e investigaciones estudiadas. Debido a que se obtuvo información más allá de lo que se puede conocer a través de algún documento que refiera al tema, ya que la investigación cualitativa no está interesada en la explicación de los fenómenos, sino en la comprensión de la misma, de esta manera es que abre camino a futuras investigaciones para 
el conocimiento del sentido de continuidad que otorgan las pacientes frente a sus problemáticas.

\section{Declaración de financiamiento y de conflictos de interés}

El estudio fue financiado por los autores, quienes declaran no tener algún tipo de conflicto de interés en la investigación realizada

\section{Correspondencia:}

Bárbara Cerda Aedo

Correo electrónico:

psbcerda@gmail.com

\section{REFERENCIAS BIBLIOGRÁFICAS}

Cayupe, R. (2013). Diseño y validación de un instrumento para evaluar los tipos de organización de significado personal (OSP) predominantes [Tesis, Magíster en Psicología Clínica de Adultos, Universidad de Chile]. Repositorio Académico de la Universidad de Chile. Recuperado de: http:// repositorio.uchile.cl/handle/2250/130690

Crittenden, P. (2006), A Dynamic-Maturational Model of Attachment. Australian and New Zealand Journal of Family Therapy, 27(2), 105-115. https:// doi.org/10.1002/j.1467-8438.2006.tb00704.x

Harcha, P. (2012, December). ¿Qué estilos de apego o vínculo afectivo existen? El Espacio de Priscilla Sobre Psicología y Psicoterapia . https:// prisciharcha.wordpress.com/2012/12/05/queestilos-de-apego-o-vinculo-afectivo-existen/

Krause, M. (1995). La investigación cualitativa: un campo de posibilidades y desafíos. Temas de Educación, (7), 19-40.

León, A., y Tamayo, D. (2011). La psicoterapia cognitiva posracionalista: un modelo de intervención centrado en el proceso de construcción de la identidad. Katharsis, 0(12), 37-58. https://doi. org/10.25057/25005731.153
Marini, A., Moltedo, A., y Giordani, M. (2017). Organización de significado personal en la experiencia inmediata: cómo la personalidad influye en la respuesta al estrés psicosocial en un grupo de mujeres jóvenes. Panamerican Journal of Neuropsychology, 0-3. Recuperado de: http:// www.cnps.cl/index.php/cnps/article/view/275

Méndez, S. (2012). La amistad en la adolescencia :: El grupo de pares o amigos en el adolescente. Innatia. Recuperado de:http://www.innatia. $\mathrm{com} / \mathrm{s} / \mathrm{c}$-consejos-para-los-padres/a-amistad-enadolescencia.html

Naranjo, C., Gallardo, M., y Zepeda, M. (2010). Estilo afectivo y estilos de personalidad internamente orientados (Inward) y externamente orientados (Outward): modelo de estilos emocionales de personalidad. Revista Chilena de NeuroPsiquiatría, 48(4), 344-355. https://doi. org/10.4067/s0717-92272010000500009

Pena, L., y Oliveira, C. (2015). Psicoterapia de Vittorio Guidano e suas influências epistemológicas. Psicologia Clinica, 27(1), 245-265. https://doi. org/10.1590/0103-56652015000100014

Ruiz, A. (26 de octubre de 2007). La psicología y la terapia cognitiva post-racionalista: Aspectos teóricos y Clínicos [Conferencia Magistral]. XV Congreso Mexicano de Psicología, Hermosillo, México. Recuperado de: https://web.inteco.cl/ articulos/id/16

Torrente, F. y Harf, N. (1995). Nuevas perspectivas de la psicoterapia cognitiva: Posracionalismo, emoción y significado personal. Entrevista a Vittorio Guidano, Michael Mahoney, Humberto Maturana, Leslie Greenberg, Juan Balbi y Héctor Fernández Alvarez. Perspectivas Sistémicas, 8(38). Recuperado de: http://www.robertexto. com/archivo18/nuevas_persp.ht

Recibido: 21/01/2021

Aceptado: 23/05/2021 